\title{
Bilayer Graphene Membrane for Hydrogen Separation: Insights from Molecular Dynamics
}

\author{
Zhu Lei ${ }^{1,2, a)}$ \\ ${ }^{1}$ State Key Laboratory of Heavy Oil Processing, China University of Petroleum, Qingdao 266580, \\ Shandong, P. R. China \\ ${ }^{2}$ College of Science, China University of Petroleum, Qingdao 266580, Shandong, P. R. China \\ azhuleiupc@126.com
}

Keywords: Bilayer graphene, hydrogen separation, molecular dynamics simulations

Abstract. In this paper, we study the hydrogen separation performance of bilayer graphene by molecular dynamics simulations. When the interlayer spacing between two grapheme sheets is larger than $5 \AA$, the impurities gas molecules can pass through the membrane. When the interlayer spacing is $5 \AA$, the permeance of hydrogen can reach $4.2 \times 10^{-4} \mathrm{~mol} \mathrm{~s}^{-1} \mathrm{~m}^{-2} \mathrm{~Pa}^{-1}$, which is far higher than the industrial acceptable permeance. With the exceptionally hydrogen selectivity over the other gases, we can get the conclusion that the optimal interlayer spacing for hydrogen separation is $5 \AA$. These founding may be useful for designing of gas separation membrane with excellent performance.

\section{Introduction}

Since the 1980s, membrane separation technology has been become more and more popular as a commercial process on a grand scale due to its low energy cost, flexible structures and other merits. Due to gas permeability of a membrane is inversely proportional to its thickness for separation membrane, the traditional gas separation membranes, such as polymer, silica, metal-organic framework (MOF), with range from tens of nanometers to several micrometers in thickness are difficult to balance the relationship between selectivity and permeability [1, 2, 3]. Therefore, graphene and related materials it derives are considered as excellent membrane materials in gas separation applications by its nature of single-atom thickness [4]. So far, most studies have considered an idealized, single-layer system with variations in several key parameters, such as nanopore size or chemical modification [5, 6]. Zhu and his coworkers have demonstrated that $\mathrm{C}_{2} \mathrm{~N}$ monolayer with $3.0 \AA$ pore size showed an exceptionally high selectivity for $\mathrm{He} / \mathrm{Ne}\left(\mathrm{Ar}, \mathrm{CH}_{4}, \mathrm{CO}_{2}, \mathrm{~N}_{2}\right.$, et al.) in a wide range of temperature [5]. Shan et al. has investigated the separation of $\mathrm{CO}_{2}$ from a mixture of $\mathrm{CO}_{2}$ and $\mathrm{N}_{2}$ using a porous graphene membrane using molecular dynamics (MD) simulations and it was found that chemical functionalization of the graphene sheet can increase the absorption ability of $\mathrm{CO}_{2}$, while chemical functionalization of the pore rim can significantly improve the selectivity of $\mathrm{CO}_{2}$ over $\mathrm{N}_{2}$ [6].

Although great efforts have been paid to the synthesis of large scale graphene films [7], producing large area of perfect monolayer graphene remains highly challenging experimentally. In particular, chemical vapor deposition (CVD) [8], the primary method used for synthesizing graphene sheets, results multilayer coverage in substantial. At present, the gas separation performance of these multiple graphene layers membrane is largely unknown.

In this paper, taking hydrogen separation as an example, we perform molecular dynamics (MD) simulations to study the performance of gas separation for the two-dimensional (2D) bilayer graphene membrane. The interlayer spacing is considered in this work.

\section{Computational details and models}

MD simulations are implemented by the DISCOVER code in the Material Studio software. The interatomic interactions are described by the force field of a condensed-phase optimized molecular potential for atomistic simulation studies (COMPASS) [9]. All full atomistic simulations are subject to an NVT ensemble, and the temperature of the system is controlled by the Andersen thermostat method, 
with a fixed time step of $1 \mathrm{fs}$. Van der Waals interaction and Ewald electrostatic interaction are applied with a cutoff distance of $9.5 \AA$. Periodic boundary conditions are applied in the $\mathrm{x}$ and y directions.

In order to simulate the hydrogen separation performance of $2 \mathrm{D}$ bilayer graphene membrane, a simulation model was first established, as shown in Fig. 1. Two graphene sheets $(49.2 \AA \times 63.9 \AA)$ serve as bilayer graphene membrane and the carbon-carbon opening of the slit, dslit, is fixed at $8.5 \AA$, the offset of the slit is $42 \AA$. The "gas reservoir" consists of $60 \mathrm{H}_{2}$ molecules, $60 \mathrm{CH}_{4}$ molecules, 30 $\mathrm{CO}_{2}$ molecules and $30 \mathrm{CO}$ molecules. At the bottom of the permeate phase, a wall composed of helium atoms is used to impede gas molecules passing to the vacuum phase due to the periodicity along the $\mathrm{Z}$ axis.

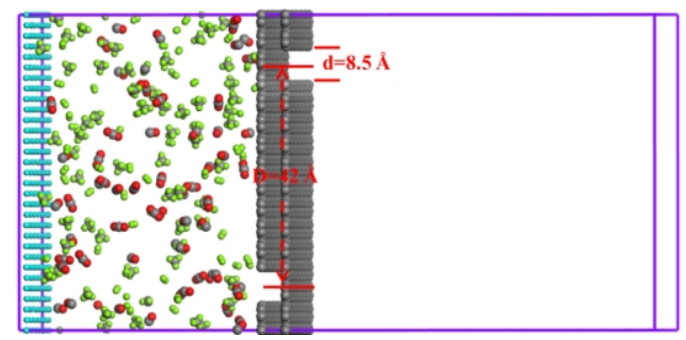

Fig. 1 The simulation model of hydrogen separation.

\section{Results and discussion}

Using the bilayer grapheme sheets with $5 \AA$ interlayer spacing as the membrane, we firstly perform the MD simulations to observe the hydrogen separation process. The snapshots of the gaseous mixture penetrating through bilayer graphene membrane in the simulation from 0 to $5 \mathrm{~ns}$ can be found in Fig. 2 . The gas separation process can be divided into three steps. First, gas molecules approach to the membrane slit. Then, the gas molecules linger on the surface near the slit for a few picoseconds before successfully crossing the barrier. Finally, hydrogen molecules go to the other side of the membrane.

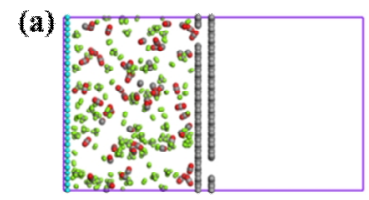

(d)

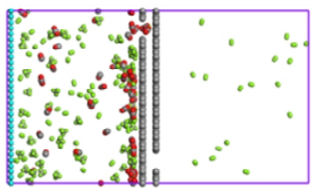

(b)

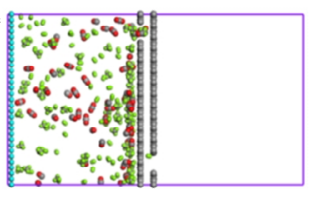

(e)

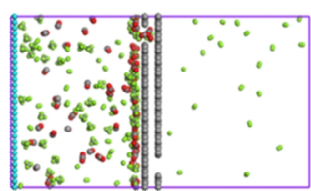

(c)

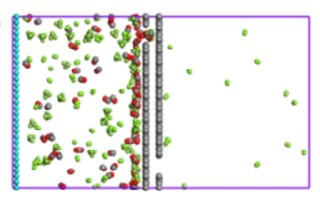

(f)

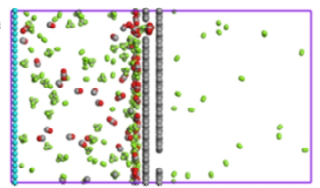

Fig. 2 Snapshots of configuration of gaseous mixture penetrating through bilayer graphene membrane at (a) $0 \mathrm{~ns}$, (b) $1 \mathrm{~ns}$, (c) $2 \mathrm{~ns}$, (d) $3 \mathrm{~ns}$, (e) $4 \mathrm{~ns}$, and (f) $5 \mathrm{~ns}$. Hydrogen, carbon, oxygen, and helium atoms are colored by green, gray, red, and turquoise blue, respectively.

Then, we perform the MD simulations to study the hydrogen separation performance for different interlayer spacing. The results can be seen in Fig. 3. We can see that when the interlayer spacing of bilayer graphene is $4 \AA$, only one hydrogen molecule pass through the membrane, and when the interlayer spacing of bilayer graphene is larger than $4 \AA$, a mass of hydrogen molecules pass through the membrane. Furthermore, when the interlayer spacing is larger than $5 \AA$, there are impurities gas molecules pass through the membrane. Then, we get the permeance and selectivity of hydrogen molecules for bilayer graphene membrane with different interlayer spacing. As shown in Fig. 4, the permeance of hydrogen is nearly zero and hydrogen molecules hardly pass through this bilayer graphene membrane as the interlayer spacing of the bilayer graphene is $4 \AA$. And when the interlayer spacing of the bilayer graphene membrane is $6 \AA$ and $8 \AA$, the hydrogen permeance can reach as high as $6 \times 10^{-4} \mathrm{~mol} \mathrm{~s}^{-1} \mathrm{~m}^{-2} \mathrm{~Pa}^{-1}$, which is far higher than the industrial acceptable value. The selectivity of hydrogen over other gas for the mixtures is defined as follows: 


$$
S_{\mathrm{H}_{2} / \mathrm{CO}_{2}}=\frac{y_{\mathrm{H}_{2}} / y_{\text {gas }}}{x_{\mathrm{H}_{2}} / x_{\text {gas }}}
$$

Where $x$ and $y$ are the mole fractions of the components in the gas phase and vacuum phase. The selectivity of hydrogen over other gases for the bilayer graphene membrane with different interlayer spacing can be seen in Table 1. When the interlayer spacing is $8 \AA$, the selectivity of $\mathrm{H}_{2} / \mathrm{CO}_{2}$ and $\mathrm{H}_{2} / \mathrm{CO}$ is 47.47 and 23.38 , respectively, which is better performance than that of other interlayer spacing, except for the $5 \AA$ interlayer spacing. And when the interlayer spacing between two graphene sheets is $5 \AA$, only hydrogen molecules can pass through the membrane, and the selectivity of hydrogen over other gases is very well. From the permeance and selectivity of hydrogen for bilayer graphene membrane with different interlayer spacing, we can get the conclusion that the optimal interlayer spacing for hydrogen separation is $5 \AA$.
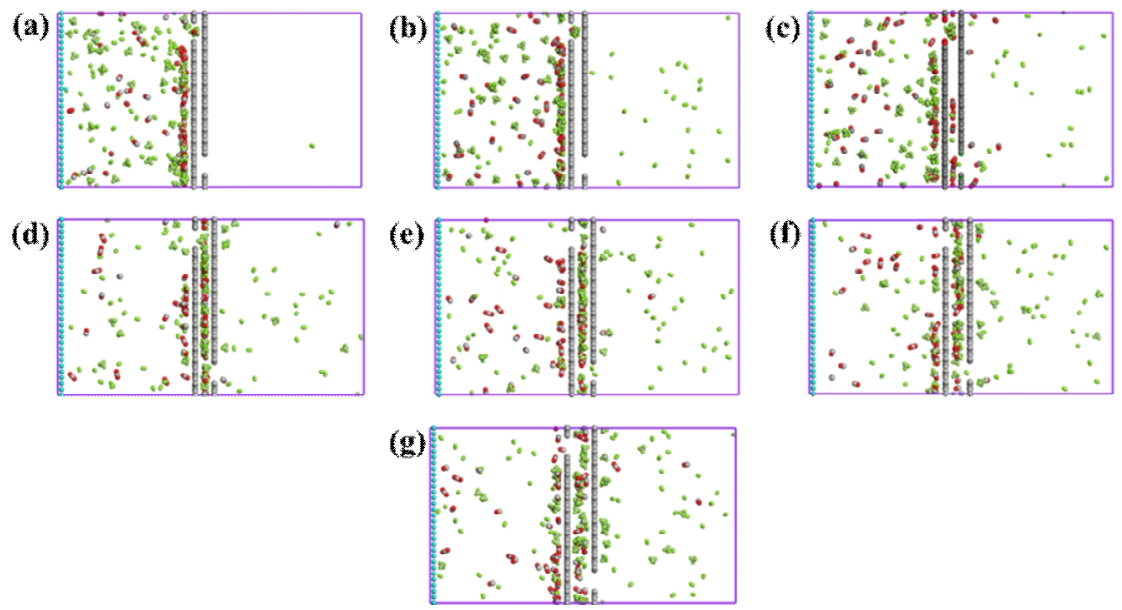

Fig. 3 The MD simulation results of the hydrogen separation for bilayer graphene membrane with different interlayer spacing, (a)-(g) the interlayer spacing of the bilayer graphene is $4 \AA$, $5 \AA, 6 \AA, 7 \AA$, $8 \AA, 9 \AA, 10 \AA$, respectively.

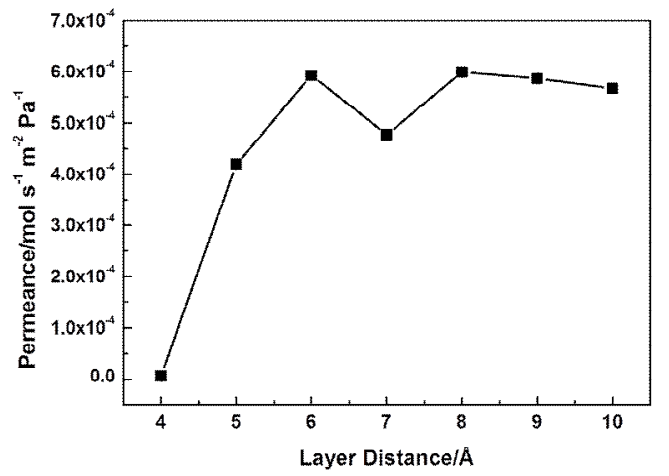

Fig. 4 The hydrogen permeance for the bilayer graphene membrane with different interlayer spacing.

Table 1. The selectivity of hydrogen over other gases for the bilayer graphene membrane with different interlayer spacing.

\begin{tabular}{cccc}
\hline Layer Distance/A & Selectivity $\left(\mathbf{H}_{\mathbf{2}} / \mathbf{C O}\right)$ & Selectivity $\left(\mathbf{H}_{\mathbf{2}} / \mathbf{C O} \mathbf{O}_{\mathbf{2}}\right)$ & Selectivity $\left(\mathbf{H}_{\mathbf{2}} / \mathbf{C H} \mathbf{H}_{\mathbf{4}}\right)$ \\
\hline 5 & $\infty$ & $\infty$ & $\infty$ \\
6 & 5.47 & 13.33 & $\infty$ \\
7 & 8.33 & 75.76 & 5.35 \\
8 & 46.76 & 94.94 & 5.22 \\
9 & 7.67 & 30.67 & 3.54 \\
10 & 8.08 & 22.31 & 2.78 \\
\hline
\end{tabular}




\section{Conclusions}

With the MD simulations, we study the hydrogen separation performance of bilayer graphene by molecular dynamics simulations. When the interlayer spacing between two grapheme sheets is larger than $5 \AA$, the impurities gas molecules can pass through the membrane. When the interlayer spacing is $5 \AA$, the permeance of hydrogen can reach $4.2 \times 10^{-4} \mathrm{~mol} \mathrm{~s}^{-1} \mathrm{~m}^{-2} \mathrm{~Pa}^{-1}$, which is far higher than the industrial acceptable permeance. With the exceptionally hydrogen selectivity over the other gases, we can get the conclusion that the optimal interlayer spacing for hydrogen separation is $5 \AA$.

\section{Acknowledgements}

This work is supported by the Natural Science Foundation of China (11374372 , 41330313), Taishan Scholar Foundation (ts20130929), the Fundamental Research Funds for the Central Universities (15CX06076A, 16CX06024A), Graduate Innovation Fund of China University of Petroleum (YCXJ2016078).

\section{References}

[1] L.M. Robeson: J. Membrane Sci. 320 (2008) 390-400.

[2] L. Zhu, Q. Xue and X. Li: ACS Appl. Mater. Inter. 7 (2015) 28502-28507.

[3] E.S. Peterson and M.L. Stone: J. Membrane Sci. 86 (1994) 57-65.

[4] J.C. Meyer, A.K. Geim and M.I. Katsnelson: Nature, 446 (2007) 60-63.

[5] L. Zhu, Q. Xue and X. Li: J. Mater. Chem. A, 3 (2015) 21351-21356.

[6] M. Shan, Q. Xue and N. Jing: Nanoscale, 4 (2012) 5477-5482.

[7] A. Reina, X. Jia and J. Ho: Nano Lett. 9 (2008) 30-35.

[8] Y. Hao, M.S. Bharathi and L. Wang: Science, 342 (2013) 720-723.

[9] M.S. Shafeeyan, W.M.A.W. Daud and A. Houshmand: Appl. Surf. Sci. 257 (2011) 3936-3942. 